\title{
Brucellar spondylitis mimicking lumbar disc herniation. Case report
}

\author{
T Kılıç ${ }^{1}$, A Fahir Özer ${ }^{2}$, S Özgen ${ }^{1}$ and M Necmettin Pamir ${ }^{3}$ \\ ${ }^{1}$ Resident, ${ }^{2}$ Associate Professor, ${ }^{3}$ Professor and Chairman, Marmara University Hospital, Department of \\ Neurosurgery, Tophanelioğlu Caddesi No. 13-15, Altunizade, Istanbul 81190, Turkey
}

\begin{abstract}
In this paper, a patient with brucellar spondylitis who was initially diagnosed as having a lumbar disc herniation is presented. As the disc tissue enlarges in the early stages of discitis because of the inflammatory reaction, it may compress the related root(s) mimicking a disc herniation. For this reason infectious discitis, as in our case, should be kept in mind in the differential diagnosis of the radicular symptoms caused by a disc herniation. The crucial role of magnetic resonance imaging in making the differential diagnosis of the case is especially emphasized.
\end{abstract}

Keywords: brucellosis; spondilitis; lumbar disc herniation; MRI study

\section{Introduction}

Brucellosis is caused by an aerobic, non-encapsulated, non-motile, Gram-negative coccobacillus, which is commonly found in domestic animals and is transmitted to humans by either direct contact or by ingestion of unpasteurized milk and dairy products. ${ }^{1-3}$ Human brucellosis is primarily an occupation related disease and affects approximately 500000 people per year worldwide. ${ }^{3}$

Brucellar spondylitis is among the most important complications of the zoonosis. The spine is the most common site of skeletal brucellosis, the lower lumber region being the most frequently affected site. ${ }^{4}$

The spinal form of brucellosis has no specific symtomatology or clinical finding, therefore a high index of suspicion is necessary for the correct early diagnosis. Low back pain with or without leg pain is the most common symptom of spinal brucellosis, and may be accompanied by radiculopathy, a positive straight leg-raising test, and paravertebral muscle spasm, making the differential diagnosis even more challenging. ${ }^{3}$

In this paper, a patient with brucellar spondylitis who was initially diagnosed as having a lumbar disc herniation is presented. The crucial role of magnetic resonance imaging (MRI) in making the differential diagnosis of the case is especially emphasized.

\section{Case report}

The patient was a 25-year-old female and her major complaint was low back pain lasting for 2 years and left leg pain for 4 months. She did not benefit from 2 months of conservative treatment including physiotherapy. The physical examination revealed no motor loss and hypoaesthesia was present in the $\mathrm{S} 1$ dermatome. The straight leg raising sign was positive at 30 degrees on the left site. The deep tendon reflexes were normal. Routine laboratory evaluation including hepatic function tests and alkaline phosphatase were all within normal limits. The erythrocyte sedimenta- tion rate, calculated by the Westergren method, was $30 \mathrm{~mm} \mathrm{~h}^{-1}$. Acute phase reactants, CRP, RF and ASO were all negative. PPD skin test was negative and her chest radiograph was normal.

Direct X-ray investigation revealed sacralization of the L5 vertebra, and a diffuse osteoporotic appearance of spinal bony structures.

Lumbar computed tomography (CT) and myelo-CT investigations revealed dural sac compression at the L5-S1 level by the bulging intervertebral disc (Figure 1). Electromyography (EMG) showed chronic denervation of the muscles innervated by the left L5 nerve root.

MRI examination revealed decreased signal intensity in the L5-S1 intervertebral disc; the L5 vertebra corpus was hypointense and T1- and hyperintense in T2-weighted sections. Indentations and lytic lesions were determined in the inferior and superior end-plates of L5 and S1 vertebral

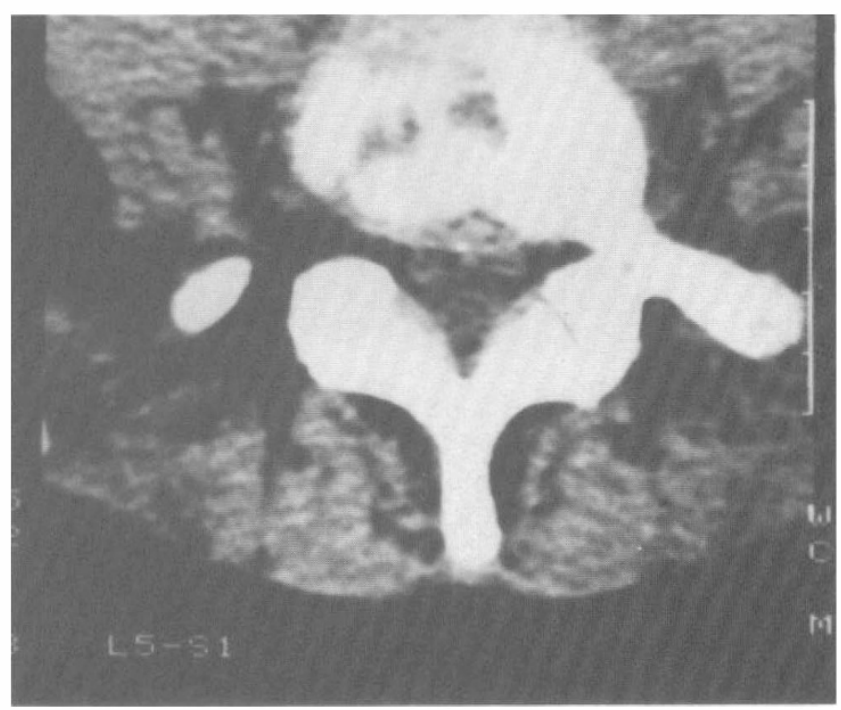

Figure 1 Lumbar CT scan revealed dural sac compression at the L5-S1 level by the enlarged intervertebral disc tissue due to the infectious process 
bodies, respectively. Bilaterally roots were minimally compressed at L5 and S1 levels. Minimal para- and prevertebral soft tissue components were found to be hyperintensified. MR findings were compatible with the diagnosis of L5-S1 discitis and osteomyelitis (Figure 2).

No fever was observed during the patient's hospital stay and no microorganism was cultured from blood samples.

Standard tube agglutination (STA) test, quantifying serum IgG level against Brucella antigen was titred as $1 / 320$, which was twice the normal dilution and the brucella Wright test was positive.

Since MRI demonstrated early radiographic signs of osteomyelitis and spondylitis and brucella agglutination titer was higher than $1 / 160$, a diagnosis of spinal brucellosis was made and the patient was treated medically, with doxycilin $200 \mathrm{mg} \mathrm{day}^{-1}$ and rifampicin $900 \mathrm{mgday}^{-1}$. During the course of the 6 weeks' medical treatment, the agglutination titre decreased to the final dilution of $1 / 160$ and the neurological signs and symptoms of low back pain gradually resolved. Concurrently the hypointense appearance of the inflammatory reaction, on the MRI scans decreased (Figure 3).

\section{Discussion}

Brucellosis is a systemic infectious disease, usually characterized by fever of unknown origin, anorexia, lymphadenopathy, hepatosplenomegaly and, symptoms and signs related to the skeletal system. ${ }^{5,6}$ Classical undulent fever cannot be found in most cases. ${ }^{1,3}$ Between 11 and $80 \%$ of patients with brucellosis have bone and joint involvement and, of those, $6-54 \%$ have spinal column involvement ${ }^{2-5,7}$ most commonly affecting the lumbar spine. ${ }^{1,8}$ Localized spinal pain is the earliest sign of spondylitis. ${ }^{1}$ Of those with spondylitis, between 10 and $43 \%$ have some degree of

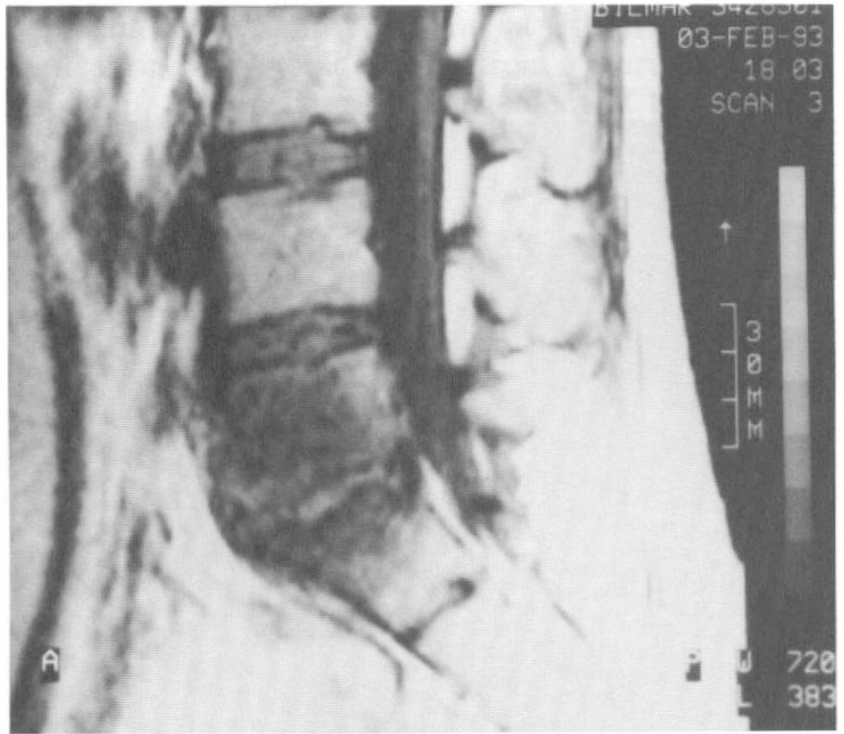

Figure 2 Lumbar MRI examination revealed decreased signal intensity in the L5-S1 intervertebral disc; L5 vertebral body was hypointense in T1-weighted sections. Indentations and lytic lesions were determined in the endplates neurological deficit ${ }^{2,6}$ and in $10-20 \%$ a paraspinal abscess develops. The spinal form of brucellosis has no specific symptomatology or clinical findings; therefore only a high degree of suspicion and a logical algorithm of differential diagnosis work-up would lead to its diagnosis.

Routine radiological investigation, including only direct radiograph and CT may not help in the differential diagnosis of the disease, and may mislead one to diagnose another cause of low back pain and/or radiculopathy, such as lumbar disc herniation, which was the case in our patient. MRI, which is a routine investigation in the work-up of patients, where surgery is thought to be indicated, was the crucial step in making the differential diagnosis. MRI revealed an intact vertebral architecture despite evidence of diffuse vetebral osteomyelitis, disc space involvement, minimal associated paraspinal soft-tissue involvement and no gibbus deformity, and supported the diagnosis of brucellar spondylitis. When compared to the other inflammatory conditions there was no characteristic finding, but it was definitely differentiated from lumbar disc herniation. Though blood cultures were all negative, serological investigation strongly supported the differential diagnosis of spinal brucellosis. The agglutination test was repeated at the end of the medical treatment period and was positive at a higher dilution. Additionally, a control MRI scan showed healing of the infected tissue without a relapse of spondylitis which is consistent with $40 \%$ relapse rate reported in the literature. ${ }^{2,3}$

In conclusion, as the disc tissue enlarges in the early stages of discitis because of the inflammatory reaction, it may compress the related root(s) mimicking a disc herniation. Therefore infectious discitis, as in our case, should be kept in mind, in the differential diagnosis of

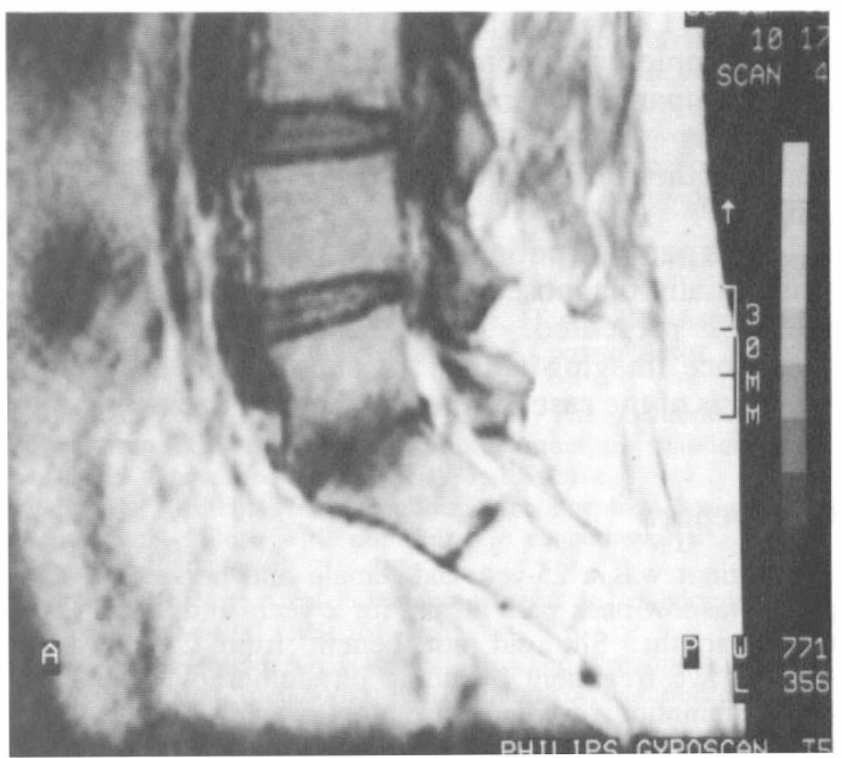

Figure 3 After 6 weeks of medical treatment, the hypointense appearance of infectious process on the T1-weighted MRI scans turned back to almost normal 
radicular symptoms caused by disc herniation. So, we state that detailed MRI studies in those with low back pain is especially valuable in the determination of the etiology.

\section{References}

1 Keenan JD, Wetz CW. Brucella spondylitis: A brief review and case report. Clin Orthop 1972; 82: 87-91.

2 Lifeso RM, Harder E, McCorkell SJ. Spinal brucellosis. J Bone
Joint Surg Br 1985; 67: 345-351.

3 Tekkök IH et al. Neurosurgery 1993; 33: 838-844.

4 Mikolich DJ, Bsyca JM. Brucella species. In: Mandell GL, Douglas RG Jr, Bennett JE (eds). Principles and Practice of Infectious Diseases. 3rd edn. Churchill Livingstone: New York, 1990, pp 1735-1742.

5 Pritchard DJ. Granulomatous infections of bones and joints. Orthop Clin North Am 1975; 6: 1029-1047.

6 Mousa ARM et al. Osteoarticular complications of brucellosis. A study of 169 cases. Rev Infect Dis 1987; 9: 531-543.

7 Ganado W, Craig AJ. Brucellosis myelopathy. J Bone Joint Surg Am 1958; 40: 1380-1388. 